\title{
Acknowledgments to Reviewers
}

The Editors thank the following reviewers, whose careful evaluations and helpful recommendations greatly assisted us during the past year and help maintain Law $\mathcal{E}^{2}$ Social Inquiry's high standards for scholarly work in sociolegal studies.

\begin{tabular}{|c|c|c|c|}
\hline Abel, Gillian & Bartholomew, & Bracewell, Lorna & abe, \\
\hline Abel, Richard & Mark & Bradford, Ben & Rosemary \\
\hline Ahmed, Shazeda & Bartlett, Katharine & Brehm, Hollie & Corrigan, Rose \\
\hline Akbar, Amna & Barzun, Charles & Brehm, Stefan & Cruz, Katie \\
\hline Aksenova, Marina & Bateman, David & Brigden, Noelle & Cushman, Barry \\
\hline Alphonso, & Beebe, Barton & Brinks, Daniel & Dancy, Geoff \\
\hline Gwendoline & Bell, Monica & Brown, Elizabeth & Das Acevedo, \\
\hline Altman, Scott & Bennett, & Burdziej, Stanislaw & Deepa \\
\hline Andersen, & Christopher & Burkhardt, Brett & Davis, Seth \\
\hline Ellen Ann & Berger, Dan & Busby, Karen & Dawe, Meghan \\
\hline Angermeyer, & Hillary & y, Shawn & ets, A.H.M. \\
\hline Philip & Berlin, Mark & ra, Gregory & \\
\hline Aragoneses, Alfons & Berrey, Ellen & iqun & a, Juan \\
\hline Atak, Kivanc & Biddulph, Sarah & Carbado, Devon & de Sa e Silva, \\
\hline Atienza, Paul & Bignami, Francesca & Carpenter, Anna & Fabio \\
\hline Atshan, Sa'ed & Bliss, John & no, Ursula & De, \\
\hline Aviram, Hadar & Bob, Clifford & t, Benjamin & Degenshein, Anya \\
\hline Azocar, María José & Boittin, Margaret & a, Moeen & Dehm, Julia \\
\hline Bagchi, Aditi & Boni-Saenz, & man, Nick & Derman, Brandon \\
\hline Baker, Kimberly & Alexander & , Weitseng & Devins, Neal \\
\hline Bali, Asli & Bosia, Michael & ton, Adam & nd, Shari \\
\hline Ballakrishnen, & Bosvieux- & Yukyong & Dias, Vitor \\
\hline Swethaa & Onyekwelu, & , Lynette & Didwania, \\
\hline Balleisen, Edward & & Clair, Matthew & \\
\hline Barkan, Joshua & Bosworth, Mary & Cohen, Mathilde & Dinovitzer, Ronit \\
\hline Barkan, Steven & Boucai, Michael & Simon & Dolovich, Sharon \\
\hline Barker, Vanessa & Boyd, Christina & ins, Paul & Dua, Jatin \\
\hline Barnes, Alison & Boyle, Elizabeth & Cooley, Will & Duffy, Mignon \\
\hline
\end{tabular}




\begin{tabular}{|c|c|c|c|}
\hline Durfee, Alesha & Gowder, Paul & Hughes, Emily & Levy, Marin \\
\hline Edmund, & Graber, Mark & Humphreys, & Li, Darryl \\
\hline Cheng Wai & Green, Tristin & Stephen & Light, Michael \\
\hline Elfenbein, Hillary & Greiner, James & Huq, Aziz & Liljeblad, Jonathan \\
\hline Ellett, Rachel & Grinvald, Leah & Husak, Douglas & Lin, Chien-Chih \\
\hline rakat, Noura & Chan & Ingram, Matthew & Logan, Wayne \\
\hline Ergas, Yasmine & Grosso, Catherine & Ip, Eric & Longhofer, Wesley \\
\hline rie, Matthew & Gulati, Mitu & Jackson, Jonathan & Longman, Tim \\
\hline scudero, Kevin & Gunneflo, Markus & Jacobi, Tonja & Lynch, Mona \\
\hline stelle Long, Doris & Gupta, Akhil & Jamar, Astrid & MacCoun, Robert \\
\hline yer, Katie & Guzik, Keith & Jenness, Valerie & Machura, Stefan \\
\hline an, M.D. & Hahm, Chaihark & Johnson, Kevin & Macias-Rojas, \\
\hline erree, Myra & Haider-Markel, & Johnson, Robert & Patrisia \\
\hline 1, Joseph & & Jones, Craig & Mack, Kenneth \\
\hline ischman, Joshua & Hajjar, Lisa & Kaplan, Paul & Madeira, Jody \\
\hline isk, Catherine & Hale, Sandra & Karekwaivanane, & Maher, Thomas \\
\hline ood, John & Haltom, William & & Mahmud, Tayyab \\
\hline lores, Andrea & Halushka, John & Kastner, Tal & Majeed, Debra \\
\hline orest, Benjamin & nerslev, Ole & tan, Sarah & ing, Jo \\
\hline oster, Sheila & $\mathrm{Ha}$ & Beth & Marder, Nancy \\
\hline rancis, Leslie & Andrew & Kenney, Sally & Margulies, Joseph \\
\hline rank, David & Craig & ssey, Jasmine & Mark, Alyx \\
\hline rankenreiter, Jens & , Lynne & Khan, Mary & Markens, Susan \\
\hline redette, Jennifer & John & gard, Mark & Marshall, \\
\hline $\mathrm{u}$, Hualing & & el, Jonathan & na-Maria \\
\hline Gallagher, Mary & redt, Karen & Kirkham, Richard & Martin Rountree, \\
\hline arcia, Beatriz & Jack & Klerman, Dan & Meredith \\
\hline Garcia, Ruben & Jeanne & Krawiec, Kimberly & Massoud, Mark \\
\hline Garth, Bryant & Katharina & Kreis, Anthony & $\mathrm{Ma}$ \\
\hline ash, Alison & recht, & ner, Roy & Goldburn \\
\hline Saskins, Richard & ney & Kreitzer, Rebecca & McCallister, \\
\hline Gervais, Daniel & Rachael & er, Tor & Jacqueline \\
\hline GhaneaBassiri, & Hironaka, Ann & Krishnan, Jayanth & McCarthy, Lauren \\
\hline Kambiz & $\mathrm{Hi}$ & er, $\mathrm{Ti}$ & McMorrow, Judith \\
\hline Gilad, Sharon & Ho, Daniel & jak Ivkovich, & Meares, Tracy \\
\hline Giles, Micheal & Hoff, & & Meernik, James \\
\hline Gill, Nick & Hoffman, Elizabeth & , Nicola & Menkel-Meadow, \\
\hline Ginsburg, Tom & Hoffman, & Lakier, Genevieve & \\
\hline Glick, David & tha & Larsen, Allison Orr & Merry, Sally \\
\hline Goldbach, Toby & Hollander, & Lazarus-Black, & Milov, Sarah \\
\hline Golder, Ben & & & Mitsilegas, \\
\hline Goldman, Shelly & Hollis-Brusky, & Lei, Ya-Wen & Valsamis \\
\hline Goldstein, Alyosha & & Lejeune, Aude & Moinester, Margo \\
\hline Goodale, Mark & Hoyos, Roman & Leon, Chrysanthi & Moore, Ilona \\
\hline Goodman, Philip & Hsu, Chin-Fang & Leverentz, Andrea & Morris, Meghan \\
\hline Gordon, Neve & Huang, Cheng-Yi & Levin, Leslie & Mosely, Erin \\
\hline
\end{tabular}




\begin{tabular}{|c|c|c|c|}
\hline Moyer, Laura & Ristroph, Alice & Shachar, Ayelet & Taylor Poppe, Emily \\
\hline Nandini Ghosh, & Ritvo, Harriet & Shamir, Ronen & Taylor, Judith \\
\hline Shrimoyee & Robinson, John & Shannon, Sarah & Taylor, Matthew \\
\hline Nelson, Stephen & Robinson, Russell & Shdaimah, Corey & Teodoro, Manny \\
\hline Ng, Kwai Hang & Rogers, Brishen & Sheppard, Eric & Thacher, David \\
\hline Nielsen, & Root, Veronica & Sherman, Nicole & Thoreson, Ryan \\
\hline Laura Beth & Rosado & Sidel, Mark & Tinkler, Justine \\
\hline O'Brien, Thomas & Marzan, Cesar & Silver, Carole & Tomassetti, Julia \\
\hline O’Donnell, Ian & Rose, Mary & Singer, Simon & Trevaskes, Susan \\
\hline Owens, Ryan & Rosenberg, Gabriel & Skinner, Scott & Tuerkheimer, \\
\hline Painter, Genevieve & Rosenbloom, & Smith, William & Deborah \\
\hline Pandya, Sachin & Rachel E. & Socia, Kelly & Tyler, Tom \\
\hline Parvez, Fareen & Rubin, Ashley & Solomon, Ray & Valdivia, Carolina \\
\hline Pedriana, Nicholas & Rudrappa, & Sommerlad, Hilary & VanderVelde, Lea \\
\hline Perez, Joanna & Sharmila & Sousa, Bill & Vargas, Robert \\
\hline Petersen, Nick & Rueda, Pablo & Spaulding, & Varsanyi, Monica \\
\hline Plys, Kristin & Rumpf, Cesraea & Norman & Vatuk, Sylvia \\
\hline Popova, Maria & Ryan, Christopher & Sterling, Joyce & Vega, Irene \\
\hline Priest, Claire & Said, Wadie & Stern, Rachel & Versteeg, Mila \\
\hline Provine, & Salamone, Michael & Stevens, Jacqueline & Wald, Eli \\
\hline Doris (Marie) & Sample, Lisa & Stitt, Mary Ellen & Ward, Artemus \\
\hline Pruce, Joel & Sanchez- & Stolzenberg, Nomi & Weidemaier, Mark \\
\hline Purser, Gretchen & Urribarri, Raul & Su, Anna & Weinberg, Jill \\
\hline Quraishi-Landes, & Savelsberg, & Sullivan, Barry & Welsh, Megan \\
\hline Asifa & Joachim & Sullivan, Esther & White, Ariel \\
\hline Rabban, David & Sawyer, Logan & Sullivan, Gavin & Wilkins, David \\
\hline Read, Benjamin & Schauer, Frederick & Sullivan, Winnifred & Wilson, Joshua \\
\hline Reinert, Alexander & Schein, Louisa & Sumner, Jennifer & Woeste, Victoria \\
\hline Reiss, Dorit & Schlanger, Margo & Sundstrom, Lisa & Woodson, Kevin \\
\hline Renteln, Alison & Schlesinger, Traci & Swiss, Liam & Young, Jacob \\
\hline Dundes & Scott, Elizabeth & Taite, Phyllis & Zatz, Marjorie \\
\hline Rhode, Deborah & Sevier, Justin & Tanenhaus, David & Zimmerman, Arely \\
\hline Rider, Chris & Sezgin, Yuksel & Tankebe, Justice & Zimring, Franklin \\
\hline
\end{tabular}

